\title{
Pie risk, una herramienta para la prevención del pie diabético
}

\author{
Arturo Orduz $z^{1}$, Claudia Tique ${ }^{2}$, Ingrid Stetphens ${ }^{3}$, Andrés González $z^{4}$ \\ Barengo Noel ${ }^{5}$ Diana Tamayo ${ }^{6}$
}

\begin{abstract}
${ }^{1}$ Sección de endocrinología, departamento de medicina interna, jefatura Clínica de pie Diabético y Salvamento de la extremidad, Fundación Santa Fe de Bogotá. Bogotá, Colombia.

${ }^{2}$ Sección de endocrinología, departamento de medicina interna, enfermera jefe, educadora en diabetes, Fundación Santa Fe de Bogotá. Bogotá, Colombia.

${ }^{3}$ Sección de endocrinología, departamento de medicina interna, Internista- endocrinóloga, Directora de Island Diabetes Center, San Andrés Islas.

${ }^{4}$ Instituto para la Evaluación de la Calidad y Atención en Salud IECAS. Bogotá, Colombia.

${ }^{5}$ Consultor Observatorio de Diabetes, Colombia, Magíster y Doctor en salud pública, profesor Universidad del Tolima.

${ }^{6}$ Coordinadora Observatorio de Diabetes, Colombia, epidemióloga. Md. MSc.
\end{abstract}

Autor para correspondencia: Arturo Orduz. Correo electrónico: aorduz@cable.net.co, arturoorduzl@hotmail.com

Conflicto de interés: Este estudio no fue patrocinado por la industria farmacéutica, se realizó bajo las normas técnicas, legales sin interés diferente al conocimiento.

Fecha de recepción: 17/01/2015

Fecha de aceptación: 3/02/2016

\section{Resumen}

Introducción: El pie diabético es un problema de salud pública debido a su elevada frecuencia, severidad y a su impacto económico. En la etapa prepatogénica, el pie presenta alteraciones funcionales y estructurales que de no detectarse a tiempo pueden progresar a la úlcera. Sin embargo, el examen de los pies no se realiza en la mayoría de las consultas. La prevención primaria y la detección oportuna deben tener lugar en el primer nivel de atención.

Objetivo: Desarrollar y validar un instrumento simple y práctico para clasificar de manera temprana el pie del paciente diabético en riesgo por parte de la enfermera entrenada antes de la visita a su médico tratante, teniendo en cuenta todos los elementos patogénicos.

Métodos: Estudio transversal. Se creó una escala a partir de la experiencia clínica en donde se califican ocho factores de riesgo para desarrollar ulceración: tiempo de diabetes desde el diagnóstico, control metabólico, presencia de síntomas de neuropatía diabética, diagnóstico de la neuropatía por monofilamento, presencia de claudicación intermitente y ausencia de pulsos periféricos, presencia de deformidades, presencia de infecciones locales e historia anterior de úlceras que sanaron, amputaciones menores o pie de Charcot. Los puntajes fueron asignados según gravedad. Esta herramienta fue aplicada a un grupo de 204 diabéticos con ulceración y 207 pacientes diabéticos sin ulceración.

Resultados: El promedio de edad fue de 65 años, el 42,8\% de los pacientes eran hombres. La edad y todos los ítems de la escala, excepto hemoglobina glucosilada, fueron asociados con la presencia de úlcera. Usando un punto de corte de 10 puntos, la herramienta tuvo una sensibilidad del $100 \%$ y una especificidad del 73,4\% para el diagnóstico de úlcera, con un área bajo la curva ROC de 0,992(IC 95\%, 0,986 A 0,998).

Conclusión: La herramienta "pie risk" es útil para la calificación y valoración del riesgo de pie diabético.

Palabras clave: pie diabético, evaluación del riesgo, prevención primaria, enfermera entrenada, equipo de atención en salud.

\section{Abstract}

Background: The diabetic foot is a public health problem due to its frequency, severity and economic impact. In prepathogenic phases, the foot presents structural and functional alterations that, if not detected opportunely, will progress to an ulcer. However, a thorough examination of the diabetic patient's feet is often eluded in routine health controls.

objective: To develop and validate a simple and practical instrument for early classification of the diabetic foot risk that can be easily applied by a trained nurse before the medical visit.

Methods: Cross-sectional study. Based on clinical expertise, a scale with eight risk factors for foot ulcer was developed: time from diagnosis, glycosylated hemoglobin, neuropathy symptoms, monofilament test, absent pulses or claudication; deformities, local infection and history of either amputation, ulceration or Charcot foot. Item scores were assigned based on severity. The tool was tested in a group of 204 ulcerated diabetic patients and 207 of non-ulcerated diabetic patients.

Results: Median age was 65 years, $42.8 \%$ of patients were male. Age and all scale items except glycosylated hemoglobin 
were statically associated with ulcer presence. Using the proposed cut point of 10 points, the scale has 100\% sensitivity and 73.4\% specificity for ulcer diagnosis, with an area under the ROC curve of 0.992 (95\% CI 0.986 to 0.998).

Conclusions: 'Pie risk' is a useful tool for the evaluation and risk assessment of the diabetic patient.

Keywords: Diabetic Foot; Risk Assessment; Primary Prevention; Nurse Practitioners; Patient Care Team.

\section{Introducción}

La diabetes mellitus es un problema de salud pública mundial. El séptimo Atlas de la FID (Federación Internacional de Diabetes) reporta que hay 415 millones de diabéticos en el mundo, con la posibilidad de una tendencia ascendente y, por lo tanto, de que aumenten la cantidad de personas con complicaciones crónicas $^{(4)}$. Según los datos de la Organización Mundial de la Salud (OMS) en el 2014, la prevalencia mundial fue del 9\% entre los adultos mayores de 18 años $^{(8)}$. Actualmente la prevalencia en Colombia está alrededor del 7\% y según proyecciones, la diabetes será la séptima causa de mortalidad en el 2030, especialmente en países de ingresos bajos y medios ${ }^{(12)}$. La diabetes mellitus es una enfermedad degenerativa en donde los pacientes viven asintomáticos hasta que la hiperglucemia en el tiempo desencadena alteraciones vasculares, neurológicas, renales, retinales y cardiacas $^{(11)}$. Estudios como DCCT- EDIC en diabéticos tipo 1 y UKPDS y STENO-2, en diabéticos tipo 2, demostraron que mientras más cerca de la normalidad y por tiempos más prolongados esté el nivel deglucemia menor es la posibilidad de presentar secuelas vasculares y neurológicas ${ }^{(2-3)}$. Por lo tanto, el tiempo desde el diagnóstico y el control metabólico son factores determinantes para el desarrollo de complicaciones crónicas.

Dentro de las complicaciones crónicas, el pie diabético es uno de los problemas más frecuentes y devastadores. Se define como la presencia de ulceración en los pies. Es ocasionada por múltiples factores neurológicos, vasculares, infecciosos, metabólicos, traumáticos e inmunológicos. Es un problema de salud pública por su alto costo y magnitud. Se presenta en un $25 \%$ de los pacientes en cualquier momento de la enfermedad y de éstos el $20 \%$ terminan en amputación. La mitad de los pacientes amputados terminan perdiendo la otra extremidad en los siguientes cinco años ${ }^{(1)}$.

En su etapa prepatogénica, el pie diabético presenta trastornos estructurales y funcionales que de no detectarse continuarán su his- toria natural hasta la úlcera. Esto aumenta el riesgo de amputación, hospitalizaciones prolongadas, costos e impacto social, familiar y psicológico en el paciente ${ }^{(6)}$.

Otro problema en la atención del diabético es que sus pies son examinados en menos del $5 \%$ de las consultas. Factores como la falta de elementos diagnósticos, la ausencia de entrenamiento en el equipo de trabajo, la no presencia de síntomas claros en los pies anestesiados, el poco tiempo de atención en consulta y la indiferencia hacia un pie en riesgo, hacen que la úlcera esté presente en cualquier momento del curso de la enfermedad ${ }^{(17)}$.

Por lo tanto, analizar muy bien al paciente en la etapa prepatogénica permite tener controlada la situación y detectar lesiones estructurales o funcionales tempranas, para no permitir que el curso natural de la enfermedad termine lesionando el pie con todas sus consecuencias ${ }^{(7)}$.

\section{Métodos}

\section{Diseño}

"Pie risk" es una escala creada por expertos en pie diabético a partir de conocimiento teórico que consta de 8 puntos de interrogatorio e inspección del pie: 1- Tiempo de evolución de la diabetes en años, 2- Grado de control metabólico por última HbA1c, 3- Síntomas de neuropatía diabética como dolor quemadura, dolor cortante, adormecimiento y sensación de parestesias, 4- Examen clínico de la neuropatía con monofilamento, 5- Síntomas de claudicación y presencia de pulsos periféricos, 6- Presencia de deformidades evidentes, 7- Presencia de infección local y 8- Antecedente en el pie de ulceración, amputación o Charcot. Cada ítem, excepto el último, es calificado según la gravedad del signo o síntoma con puntajes de 1, 2 o 3 . La sumatoria total se interpreta como riesgo bajo, medio y alto de presentar pie diabético según puntos de corte teóricos (figura 1).

\section{Figura 1. Herramienta Pie Risk}

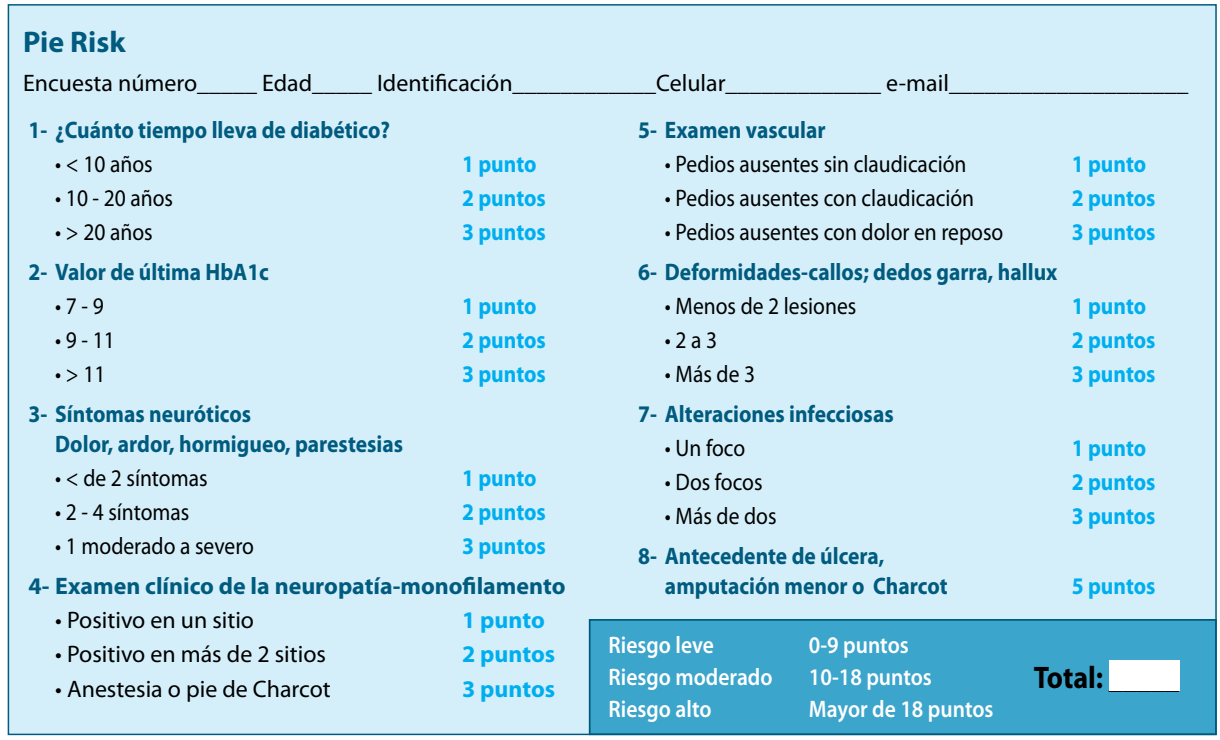


En este estudio transversal se aplicó la escala Pie Risk a 411 pacientes diabéticos divididos en dos grupos: Grupo A, 204 pacientes con diagnóstico de pie diabético con diferentes grados de ulceración, en un centro especializado en la ciudad Santo Domingo (República Dominicana), entre abril 7 y 29 de 2014 y, Grupo B , 207 pacientes con diabetes y sin ulceración, en la ciudad de San Andrés Islas (Colombia) entre enero y marzo del 2014.

\section{Objetivo}

Primario:

Aplicar en dos grupos de pacientes diabéticos, sin y con úlcera (pie diabético), la herramienta "Pie Risk", y validarla epidemiológicamente.

Secundario:

1-Categorizar el grado de riesgo del pie sin lesión.

2-Realizar educación y prevención del pie en riesgo.

3-Entrenar al grupo de enfermería en cuidados y prevención del pie como parte del equipo de atención en diabetes.

\section{Pacientes}

Se evaluaron 411 pacientes con diabetes de dos centros de atención especializada en atención de la diabetes, 204 con úlcera por pie diabético del centro INDEN (Instituto Nacional de Diabetes Endocrinología y Nutrición), Santo Domingo-República Dominicana, y 207 sin úlcera del centro Island Diabetes Center, San Andrés (Colombia). Se tomaron pacientes entre 18 y 80 años de edad que presentaran diagnóstico de diabetes tipo $2 \sin$ importar el tiempo de evolución, ni el grado de control metabólico. Para el grupo A debían tener úlcera en sus pies diagnosticada como pie diabético. Para el grupo B, sin ulceración.

\section{Procedimiento}

En la primera visita, una vez confirmados los criterios de inclusión y exclusión, se procedió a leer, explicar y firmar el consentimiento informado para, en una segunda visita, realizar el interrogatorio y el examen del pie. A todos se les aplicó la herramienta en estudio "Pie Risk", que consta de un interrogatorio de 5 preguntas: tiempo de diabetes, control metabólico, síntomas neurológicos, síntomas vasculares y antecedentes patológicos del pie; y 4 datos del examen físico: Monofilamento de Semmens-Weinstein, pulsos periféricos, valoración de deformidades y valoración de focos de infección.

Durante todo el proceso del estudio se trabajó intensamente en educación y en cuidados del pie, involucrando la familia y los acompañantes del paciente, en cuanto a cinco puntos importantes: 1- observación diaria de los pies en todos sus lados. 2- uso de crema humectante, que permita tener la piel elástica, pues la resequedad hace que se quiebre y origine una ulceración. 3- uso diario de media apropiada, a medida, sin rotos ni costuras. 4- calzado con suela y tacón continuo rígido, extraancho, con plantilla personalizada y en mecedora. 5- te- ner contacto continuo y directo con el equipo de atención, por medios virtuales, como celular, correo electrónico, mensajería instantánea y redes sociales para enviar fotografías en caso de alguna lesión inicial, dolor o dudas.

\section{Tiempo de diabetes}

La mayoría de los diabéticos, especialmente tipo 2, tienen un tiempo de evolución de su diabetes indeterminado, porque cuando se diagnostican ya tienen un tiempo de enfermedad asintomática y muchos de ellos se descubren por casualidad o por presencia de alguna complicación crónica. Cuando la glucosa es mayor de $126 \mathrm{mg} / \mathrm{dl}$ en ayunas (hiperglucemia), se produce una respuesta inmediata con acúmulo de aniones superóxido en la mitocondria, con alteraciones nucleares tóxicas, presencia productos finales de glucosilación avanzada, proteína cinasa c y factor nuclear Kappa Beta relacionados directamente con la inflamación y las complicaciones crónicas ${ }^{(8)}$.

\section{Control metabólico}

Mantener la glucosa pre y posprandial dentro del rango normal no sólo evita el inicio de las complicaciones micro y macrovasculares, también sostener internamente un ambiente euglucémico en el tiempo, previene las mismas. A este fenómeno se le ha llamado "memoria metabólica" o "efecto legado". Corregir la glucosa temporalmente, por lo tanto, no evita las complicaciones. La mejor estrategia entonces desde lo preventivo, es lograr rápidamente el mejor control metabólico y sostenerlo de la mejor manera en el tiempo ${ }^{(5)}$.

\section{Síntomas neurológicos}

La neuropatía diabética, tipo polineuropatía sensitivomotora, distal y simétrica es la complicación más frecuente y el mayor determinante para la presencia de úlcera. Se encuentra en el $80 \%$ de los pacientes y puede ser asintomática o presentar síntomas crónicos de diferentes características de acuerdo con las fibras comprometidas. Cuando hay síntomas son muy variables en intensidad y frecuencia. Para el estudio se interrogó el dolor mediante el TSS (Total Simptoms Score), herramienta utilizada en la mayoría de estudios neurológicos. El TSS considera cuatro tipos de síntomas: dolor lancinante o cortante, dolor tipo ardor quemante, parestesias y adormecimiento. Con intensidad y frecuencia determinada ${ }^{(14)}$.

\section{Examen con el monofilamento}

El monofilamento de Semmens Weinstein de 10 gramos es el estándar de detección de alteración de fibras cortas de sensibilidad, que son las que se lesionan primero. Para el estudio se evaluó inicialmente en las manos del paciente, indicándole lo que debía sentir. Luego se colocó en cuatro puntos de los pies: pulpejo del primer artejo, cabeza de primero, tercer y quinto metatarsiano. Se consideró anestesia cuando había dos puntos anormales ${ }^{(16)}$.

\section{Presencia de claudicación intermitente y palpación de pulsos periféricos}

La clasificación de Fontaine se utilizó como parámetro de evaluación del síntoma vascular, tipo claudicación intermiten- 
te, descartando otras causas de dolor lumbar o en miembros inferiores. El patrón más común en diabéticos es la alteración de la tibial anterior, peroneal y/o tibial posterior. El dolor tipo cansancio muscular, localizado o irradiado, en reposo o relacionado con la caminada en un lapso de distancia, constituyó la base del interrogatorio. Se asoció al examen físico por la presencia o no de pulsos pedios, previo entrenamiento de la enfermera y bajo su criterio. Resulta fundamental por la gran importancia del manejo de la isquemia como factor determinante para la formación de úlceras o para su curación futura ${ }^{(18)}$.

\section{Presencia de deformidades}

El roce de la piel, cuando existe una deformidad como: hallux, dedos en garra, dedos en martillo, helomas, cabalgamiento de dedos, caída o elevación de arcos, prominencias óseas, alteración de puntos de apoyo o cualquier condición de presión plantar, determina la formación de callosidades que luego se rompen y forman la ulceración. En esta condición se calificó el "Pie Risk" por el número de alteraciones ortopédicas por cada pie, asumiendo que entre más lesiones existan, más posibilidad hay de lesión ${ }^{(20)}$.

\section{Presencia de infecciones}

El pie del diabético, debido a la neuropatía autonómica, es generalmente reseco y esto hace que se presente ruptura de la epidermis con posibilidad de heridas, factibles de infección. Sin embargo, en el área interdigital con la humedad es común la presencia de micosis por candidiasis, así como también las onicomicosis por diferentes gérmenes. La onicogrifosis asociada y la alteración en la presión plantar también puede dar origen a la onicocriptosis. Todas estas infecciones oportunistas pueden cursar crónicamente de manera asintomática o pueden sobreinfectarse agudamente por bacterias con cuadros de supuración, dolor, rubor, edema o inflamación localizada. En el estudio se determinó el número de focos de infección por cada miembro ${ }^{(21)}$.

\section{Antecedente de amputación menor, pie de Charcot o úlcera curada}

La presencia de un antecedente de amputación, de un artejo o de un rayo, cambia la posición anatómica al caminar y produce un disbalance ortopédico en las presiones plantares, que alteran los puntos de apoyo, dando origen a callosidades. Así mismo, el paciente que tiene un antecedente de pie de Charcot que ha sido manejado con inmovilización, queda sensible a reincidir en el daño neuropático y hace deformidades óseas futuras, que pueden generar fenómenos de presión anormales, asociados con la presencia de úlceras. De igual manera, las úlceras curadas, sobre la base de una neuropatía diabética o una enfermedad arterial periférica, pueden reincidir de una manera más drástica, especialmente asociándose a sobreinfección bacteriana. Estas consideraciones hacen que el riesgo relativo de ulceración sea mayor en caso de amputaciones menores, antecedente de pie de Charcot o úlceras que sanaron. Se consideró en el instrumento "Pie Risk", el mayor puntaje de riesgo de lesión a estas alteraciones ${ }^{(22)}$.

\section{Análisis estadístico}

Las características clínicas básicas de la muestra se describieron usando frecuencias absolutas y relativas, así como medidas de tendencia central y dispersión.

Para cada ítem de la escala se calculó su asociación con la presencia de úlcera mediante odds ratio (OR) y sus intervalos de confianza de 95\%, usando regresión logística. Los ítems fueron tratados como variables ordinales, considerándose un puntaje de uno como el menor riesgo. El puntaje total de la escala se analizó como una prueba diagnóstica tomando como estándar de oro la presencia de úlcera en el examen físico, calculando la curva de receptor-operador (ROC) así como los valores de sensibilidad, especificidad y valores predictores para el punto de corte teorizado.

\section{Aspectos éticos}

Todos los pacientes fueron informados acerca de las características del estudio y firmaron el consentimiento informado. El análisis estadístico fue realizado de manera anónima y todos los procedimientos fueron dirigidos basados en la declaración de Helsinki.

\section{Resultados}

El promedio de edad para el grupo A fue de 68 años y para el grupo B de 63 años, total promedio 65 años.

El tiempo menor de 10 años de evolución de la diabetes en el grupo A fue de 37 pacientes (18,1\%), grupo B, 74 (37,5\%), total 111 (27\%). Entre 10 y 20 años, para el grupo A 129 pacientes (18,1\%), grupo B 124 (29,9\%), total 247 (60,1\%). Más de 20 años de evolución de la diabetes, para el grupo A, 44 pacientes (21,6\%), grupo B 9 (4,35\%), total $53(12,9 \%)$.

El control metabólico se determinó como el último valor de la hemoglobina glicosilada A1c. Para HbA1c menor de $9 \%$, para el grupo A, 59 pacientes $(28,9 \%)$, para el grupo B $136(65,7 \%)$, total $195(47,4 \%)$. HbA1c entre 9 y 11\%, para el grupo A, 97 pacientes (47,5\%), grupo B, 64 (30,9\%), total $161(39,2 \%)$. HbA1c mayor de 11\%, grupo A, 47 pacientes $(23,1 \%)$, grupo B, $6(2,9 \%)$, total $53(12,9 \%)$.

En cuanto a los síntomas neurológicos, fueron negativos en el grupo A, en 31 pacientes (15,2\%), grupo B, 37 (17,9\%), total $68,(16,5 \%)$. De uno a dos síntomas en el grupo A, 84 pacientes (41,2\%), grupo B, 115 (55,6\%), total 68 (16,5\%). Entre tres y cuatro síntomas para el grupo A, 77 pacientes (37;8\%), grupo B 47 (22,7\%), total 124 (30,2\%).

Presencia de un solo síntoma pero de intensidad moderada a severa para el grupo A, 12 pacientes $(5,9 \%)$, grupo B, 8 $(3,9 \%)$, total $20(4,9 \%)$.

En cuanto el examen con el monofilamento, fue negativo en el grupo A, en 8 pacientes (3,9\%), grupo B, 52 (25,1\%), total $60(14,6 \%)$, positivo en un sitio, grupo A, 77(37,8\%), grupo B, 86 (41,6\%), total 163 (39,7\%). Positivo en más de 
dos sitios, para el grupo A, 93 (45,6\%), grupo B, $65(31,4 \%)$, total $158(38,4 \%)$. Presencia de pie anestesiado o Charcot, grupo A, $26(12,7 \%)$, grupo B, $4(1,9 \%)$, total $30(7,3 \%)$.

En cuanto al examen vascular, tenían pulsos pedios presentes en el grupo A, 163 pacientes $(79,9 \%)$, grupo B, $186(89,9 \%)$, total 349 $(84,9 \%)$. Pulsos ausentes sin claudicación para el grupo A, 15 (7,3\%), grupo B, 12 (5,8\%), total $27(6,6 \%)$. Ausencia de pulsos con presencia de claudicación, para el grupo A, 23 (11,3\%), grupo B, 7 (3,3\%), total $30(7 ; 3 \%)$. Ausencia de pulsos con dolor en reposo para el grupo A, 3 pacientes $(1,5 \%)$, grupo B, $2(1 \%)$, total $5(1,2 \%)$.

En cuanto a la presencia de lesiones o deformidades, tenían al menos una lesión en el grupo A, 2 pacientes (1\%), grupo B, $133(64,2 \%)$, total $135(32,8 \%)$. Dos a tres lesiones para el grupo A, 36 (17,6\%), grupo B, 51(26,6\%), total $87(21,2 \%)$. Presencia de más de tres lesiones o deformidades grupo A, 166 (81,4\%), grupo B, 7 $(3,4 \%)$, total $173(42,1 \%)$.

En cuanto a presencia de al menos un foco de infección en el grupo A, 27 pacientes $(13,2 \%)$, grupo B, 28 (13,5\%), total 55 (13,4\%), presencia de dos focos de infección, para el grupo A, 23 (11,3\%), grupo B, 138 (66,7\%), total 161 $(39,2 \%)$, más de dos focos de infección, para el grupo A, 153 (75\%>), grupo B, 15 (7,2\%), total $168(40,9 \%)$.

Pacientes que no tenían antecedente de amputación menor, Charcot o úlcera curada grupo B, 198 (95,7\%), grupo B,0 (0\%), total $198(95,7 \%)$ con antecedente grupo A, 204 (100\%), grupo B, $9(4,3 \%)$. Total $213(51,8 \%)$ (tabla 1).

Se calcularon en el grupo B, los Odds Ratio y los intervalos de confianza para cada ítem y la relación con úlcera: Tiempo de diabetes 10 a 20 años OR 1,98, IC 95\%(1,24-3,16), más de 20 años OR 9,77 $(4,31-22,16)$. HbA1c entre 7 y $9 \%$ OR $0,43(0,02-7,5)$, entre 9 y $11 \%$ OR $1,51(0,09$ 24,66), más del 11\% OR 7,83 (0,43-142,2). Entre uno y dos síntomas neurológicos OR 0,87(0,5$1,15)$, de dos a cuatro síntomas neurológicos $\mathrm{OR}$ 1,95(1,07-3,56), Un solo síntoma pero de moderada a alta intensidad OR $1,79(0,64-4,93)$. Con relación al monofilamento positivo en un sitio OR $5,81(2,6-13,2)$, positivo en más de dos sitios OR 9,3 (4,14-20,88), anestesia o pie de Charcot OR 42,25 (11,63-153,37). En el examen vascular, los pulsos ausentes sin claudicación presentaron un OR 1,42 $(0,64-3,13)$, pulsos ausentes con claudi-
Tabla 1 . Características demográficas y clínicas de la población

\begin{tabular}{|c|c|c|c|}
\hline & A: ulcerados & B: no ulcerados & Total \\
\hline Hombres & $90(44,1)$ & $86(41,5)$ & $176(42,8)$ \\
\hline Edad & $68(11,5)$ & $63(10)$ & $65(14)$ \\
\hline \multicolumn{4}{|l|}{ Duración de la diabetes } \\
\hline$<10$ años & $37(18,1)$ & $74(37,5)$ & $111(27)$ \\
\hline 10-20 años & $129(60,3)$ & $124(59,9)$ & $247(60,1)$ \\
\hline$>20$ años & $44(21,6)$ & $9(4,35)$ & $53(12.9)$ \\
\hline \multicolumn{4}{|l|}{ Última HbA1c } \\
\hline$<7 \%$ & $1(0,5)$ & $1(0,5)$ & $2(0,5)$ \\
\hline $7-9 \%$ & $59(28,9)$ & $136(65,7)$ & $195(47,4)$ \\
\hline $9-11 \%$ & $97(47,5)$ & $64(30,9)$ & $161(39,2)$ \\
\hline$>11 \%$ & $47(23,1)$ & $6(2,9)$ & $53(12,9)$ \\
\hline \multicolumn{4}{|l|}{ Síntomas neurológicos } \\
\hline Ninguno & $31(15,2)$ & $37(17,9)$ & $68(16,5)$ \\
\hline $1-2$ & $84(41,2)$ & $115(55,6)$ & $199(48,4)$ \\
\hline $2-4$ & $77(37,7)$ & $47(22,7)$ & $124(30,2)$ \\
\hline 1 moderado-severo & $12(5,9)$ & $8(3,9)$ & $20(4,9)$ \\
\hline \multicolumn{4}{|l|}{ Monofilamento } \\
\hline Negativo & $8(3,9)$ & $52(25,1)$ & $60(14,6)$ \\
\hline Positivo 1 sitio & $77(37,8)$ & $86(41,6)$ & $163(39,7)$ \\
\hline Positivo 2 sitios & $93(45,6)$ & $65(31,4)$ & $158(38,4)$ \\
\hline Anestesia o Charcot & $26(12,7)$ & $4(1,9)$ & $30(7,3)$ \\
\hline \multicolumn{4}{|l|}{ Examen vascular } \\
\hline Pulsos presentes & $163(79,9)$ & $186(89,9)$ & $349(84,9)$ \\
\hline Ausentes sin claudicación & $15(7,3)$ & $12(5,8)$ & $27(6,6)$ \\
\hline Ausentes con claudicación & $23(11,3)$ & $7(3,3)$ & $30(7,3)$ \\
\hline Ausentes dolor en reposo & $3(1,5)$ & $2(1)$ & $5(1,2)$ \\
\hline \multicolumn{4}{|l|}{ Lesiones o deformidades } \\
\hline Ninguna & $0(0)$ & $16(7,7)$ & $16(3,9)$ \\
\hline $1-2$ & $2(1)$ & $133(64,2)$ & $135(32,8)$ \\
\hline $2-3$ & $36(17,6)$ & $51(26,6)$ & $87(21,2)$ \\
\hline$>3$ & $166(81,4)$ & $7(3,4)$ & $173(42,1)$ \\
\hline \multicolumn{4}{|l|}{ Focos de infección } \\
\hline Ninguno & $1(0,5)$ & $26(12,6)$ & $27(6,6)$ \\
\hline 1 & $27(13,2)$ & $28(13,5)$ & $55(13,4)$ \\
\hline 2 & $23(11,3)$ & $138(66,7)$ & $161(39,2)$ \\
\hline$>2$ & $153(75)$ & $15(7,2)$ & $168(40,9)$ \\
\hline \multicolumn{4}{|c|}{ Antecedente de úlcera Charcot o ulceración } \\
\hline Ausente & $0(0)$ & $198(95,7)$ & $198(95,7)$ \\
\hline Presente & $204(100)$ & $9(4,3)$ & $213(51,8)$ \\
\hline
\end{tabular}


cación OR 3,74(1,56-8,96), pulsos ausentes con dolor en reposo OR 1,71(0,28-10,37). En las lesiones $\mathrm{y}$ deformidades para riesgo de úlcera entre dos y tres lesiones OR46,94(10,9-202,12), mayor de tres lesiones OR 1577(322,2-7717,25). En la presencia de focos de infección, un foco OR 25,7(3,17-197,9), dos focos de infección OR 4,33(0,56-33,50), más de dos focos de infección OR 265,2(33,58-2094,31). En cuanto a los resultados de antecedente de amputación menor, Charcot o ulceración previa curada los resultados fueron no calculables (tabla 2 ).

Usando un punto de corte de 10 puntos, la herramienta tuvo una sensibilidad del $100 \%$ y una especificidad del 73,4\% para el diagnóstico de úlcera, con un área bajo la curva ROC de 0,992 (IC 95\%, 0,986 a 0,998) (figura 2).

\section{Discusión}

\section{Conclusión principal}

La identificación de los factores de riesgo y la calificación del paciente prepatogénico, es importante para la prevención del pie diabético. El "Pie Risk", como un sistema de puntaje, puede ser utilizado durante la rutina médica diaria para detectar pacientes con alto riesgo de lesión. La enfermera entrenada, en un momento diferente a la consulta, aplica la encuesta, se la entrega al paciente y ésta llega al médico tratante; de tal manera que el paciente ingrese ya valorado y calificado.

\section{Comparación de los hallazgos ante la literatura actual}

Durante muchos años se ha intentado aplicar herramientas preventivas de los diferentes estudios internacionales con resultados variables. Los resultados estadísticos son similares en las variables como factor causal. Las guías NICE y RNAO(26) enfatizan en el manejo multidisciplinario e incluyen el grupo de enfermería en prevención, diagnóstico y educación. La educación en cuidados del pie es la herramienta más efectiva y ha demostrado disminuir la incidencia de pie diabético ${ }^{(27-28)}$.

\section{Explicación de los resultados}

El $60 \%$ de los pacientes estudiados tenía una diabetes entre 10 y 20 años de evolución, en general mal controlados, pues cerca del 85\% presentaba una HbA1c por encima de los límites normales. Los síntomas neurológicos tenían diferente característica e intensidad, resultando concordante con la positividad o negatividad del monofilamento en el
Tabla 2. Asociación entre elementos del instrumento y presencia de úlcera

\begin{tabular}{|c|c|c|}
\hline & Odds Ratio & $\mathrm{IC95} \%$ \\
\hline \multicolumn{3}{|l|}{ Evolución diabetes } \\
\hline$<10$ años & Referencia & \\
\hline $10-20$ años & 1,98 & $1,24-3,16$ \\
\hline$>20$ años & 9,77 & $4,31-22,16$ \\
\hline \multicolumn{3}{|l|}{ Última HbA1c } \\
\hline$<7$ & Referencia & \\
\hline $7-9$ & 0,43 & $0,02-7,05$ \\
\hline $9-11$ & 1,51 & $0,09-24,66$ \\
\hline$>11$ & 7,83 & $0,43-142,2$ \\
\hline \multicolumn{3}{|l|}{ Síntomas neurológicos } \\
\hline Ninguno & Referencia & \\
\hline $1-2$ & 0,87 & $0,5-1,15$ \\
\hline $2-4$ & 1,95 & $1,07-3,56$ \\
\hline 1 moderado o grave & 1,79 & $0,64-4,93$ \\
\hline \multicolumn{3}{|l|}{ Monofilamento } \\
\hline Negativo & Referencia & \\
\hline Positivo en un sitio & 5,81 & $2,6-13,02$ \\
\hline Positivo en $>2$ sitios & 9,3 & $4,14-20,88$ \\
\hline Anestesia o Charcot & 42,25 & $11,63-153,37$ \\
\hline \multicolumn{3}{|l|}{ Examen vascular } \\
\hline Pulsos pedios presentes & Referencia & \\
\hline Pulsos ausentes sin claudicación & 1,42 & $0,64-3,13$ \\
\hline Pulsos ausentes con claudicación & 3,74 & $1,56-8,96$ \\
\hline Pulsos ausentes y dolor en reposo & 1,71 & $0,28-10,37$ \\
\hline \multicolumn{3}{|l|}{ Lesiones o deformidades } \\
\hline Ninguna & (No calculable) & \\
\hline 1 & Referencia & \\
\hline $2-3$ & 46,94 & $10,90-202,12$ \\
\hline$>3$ & 1577 & $322,25-7717,25$ \\
\hline \multicolumn{3}{|l|}{ Focos de infección } \\
\hline Ninguno & Referencia & \\
\hline 1 & 25,07 & $3,17-197,90$ \\
\hline 2 & 4,33 & $0,56-33,50$ \\
\hline$>2$ & 265,2 & $33,58-2094,31$ \\
\hline \multicolumn{3}{|c|}{ Antecedente de úlcera, Charcot o amputación } \\
\hline $\begin{array}{l}\text { Ausente } \\
\text { Presente }\end{array}$ & $\begin{array}{c}\text { Referencia } \\
\text { (No calculable) }\end{array}$ & \\
\hline
\end{tabular}

examen físico, siendo la patología encontrada más frecuentemente. La enfermedad arterial periférica se presentó en el 15\%. En cuanto a las deformidades, estaban presentes en el $90 \%$ de los pacientes, especialmente asociadas a neuropatía sensitiva, lo que explica la facilidad con 
Figura 2. Curva ROC

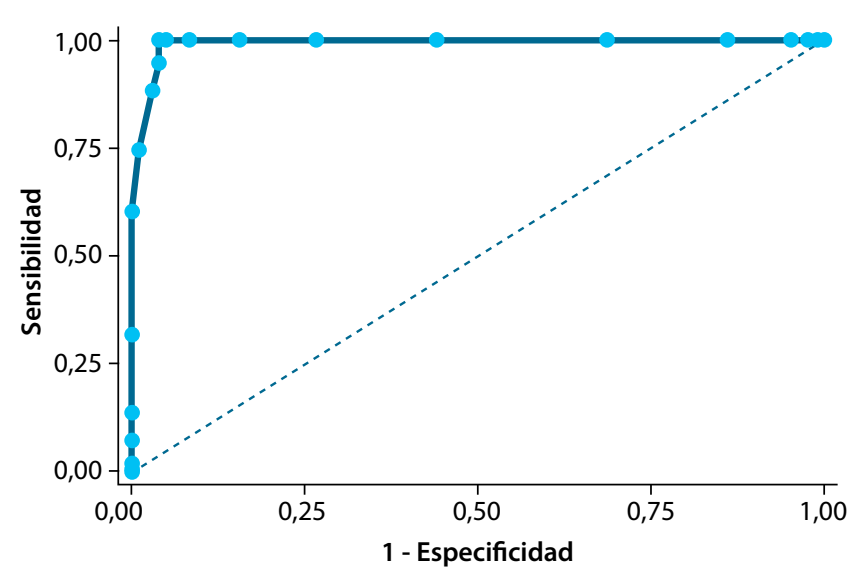

que las personas se ulceran, y más teniendo en cuenta que el $75 \%$ tenía entre 2 y 3 focos de infección sin diagnosticar. La cantidad de pacientes con antecedente de amputación, úlcera curada o Charcot fue mucho menor, con una incidencia baja, pero casi todos los pacientes reincidieron con úlcera nueva.

Se midió también la posibilidad de ulceración, encontrando cómo el tiempo de diabetes, la positividad con el monofilamento, la presencia de enfermedad arterial, las deformidades y los que tenían más de dos focos de infección presentaron un odds ratio más elevado.

\section{Fortalezas y debilidades}

El estudio tiene como fortaleza su tamaño de muestra y la aplicación del instrumento de forma estandarizada por un evaluador experimentado. Entre las debilidades se encuentran el origen diferente de los grupos de comparación y el diseño utilizado, siendo el ideal una cohorte de validación.

\section{Implicaciones en la práctica clínica y la investigación}

Es posible que los pacientes calificados con alto riesgo nunca desarrollen úlcera, debido a las medidas preventivas aplicadas. Lo importante es la detección, el diagnóstico y la corrección de los factores de riesgo. Algunos sistemas proporcionan y aplican estrategias en la contención del problema, específicamente para limitar el daño, y descuidan la prevención.

El permitir examinar a toda la población de diabéticos con el apoyo de la enfermera entrenada, en un tiempo extraconsulta, es positivo en cuanto las estadísticas demuestran que muy pocos pacientes son examinados en sus pies.

\section{Conclusiones}

La cantidad de personas con diabetes aumentará y con ello las complicaciones crónicas. El pie del diabético progresará de la etapa prepatogénica hacia la úlcera. La herramienta "Pie Risk" es una manera sencilla y rápida de categorizar el grado de riesgo en los pacientes diabéticos sin lesión.

La prevención en pie diabético y el examen periódico serán siempre los parámetros más importantes para disminuir la alta incidencia de lesiones del pie y las amputaciones. La enfermera entrenada en pie diabético es una buena alternativa y es parte del equipo multidisciplinario. Todos los pacientes son evaluados en forma integral. El lograr procesos concretos genera satisfacción en el paciente, la familia y el equipo de atención.

\section{Agradecimientos}

Este estudio se realizó en dos centros especializados en pie diabético, INDEN en Santo Domingo e Island Diabetes Center en la isla de San Andrés, con la colaboración del grupo de enfermería de dichas entidades.

\section{Referencias}

1. International Consensus on the diabetic foot. Interactive version by the international Working group on the diabetic foot. A consultative section of the International Diabetes Federation 2011, Available from : http:// www. iwgdf.org.

2. The Diabetes Control and Complications Trial Research Group. The effect of intensive treatment of diabetes on the development and progression of long term complications in insulin dependent diabetes mellitus. N Engle J Med, 1993;329;977-86.

3. United Kingdom Prospective Diabetes Study Group. Intensive blood glucose control with sulphonylureas or insulin compared with conventional treatment and risk of complications in patients with type 2 diabetes. Lancet 1998;352:837-53.

4. Aschner et al, Guias ALAD de diagnostico, control y tratamiento de la diabetes y sus complicaciones. Revista ALAS 2006; 4(3):1-78. Med 1994 : 11-166-169.

5. Aschner PJ, Ruiz AJ, Metabolic memory for vascular disease in Diabetes. Diabetes thecnol.Ther, 2012 Jun; 14 Suppl 1:s68-74.doi:10.1089/ dia.2012.0012.

6. Ragnarson Tennvall G, Apelqvist J. Health-related quality of life in patients with diabetes mellitus and foot ulcers. J Diabetes Complications [Internet]. 2000 Sep [cited 2015 Aug 26];14(5):235-41. Available from: http://linkinghub.elsevier.com/retrieve/pii/S1056872700001331.

7. Demet K, Martinet N, Guillemin F, Paysant J, André J-M. Health related quality of life and related factors in 539 persons with amputation of upper and lower limb. Disabil Rehabil [Internet]. Taylor \& Francis; 2003 Jan 11 [cited 2015 Aug 26];25(9):480-6. Available from: http://www.tandfonline.com/ doi/full/10.1080/0963828031000090434.

8. Gilbert MP. Screening and Treatment by the Primary Care Provider of Common Diabetes Complications. Med Clin North Am [Internet]. 2015 Jan;99(1):201-19. Available from: http://linkinghub.elsevier.com/retrieve/pii/S0025712514001503.

9. McCulloch DK. Evaluation of the diabetic foot. In: Post TW, editor. UpToDate [Internet]. Waltham, MA; 2014. Available from: http://www.uptodate.com/ contents/evaluation-of-the-diabetic-foot.

10. Weintrob AC, Sexton DJ. Clinical manifestations, diagnosis, and management of diabetic infections of the lower extremities. In: Post TW, editor UpToDate [Internet]. Waltham, MA; 2014. Available from: http://www.uptodate.com/contents/clinical-manifestations-diagnosis-and-managementof-diabetic-infections-of-the-lower-extremities. 
11. Alayón AN, Altamar-López D, Banquez-Buelvas C, Barrios-López K. Complicaciones crónicas, hipertensión y obesidad en pacientes diabéticos en Cartagena, Colombia. Rev Salud Pública (Bogota) [Internet]. 2009 Dec [cited 2015 Aug 18];11(6):857-64. Available from: http://www.ncbi.nlm.nih. gov/pubmed/20379659.

12. Villegas Perrasse A, Abad SB, Faciolince S, Hernández N, Maya C, Parra L, et al. El control de la diabetes mellitus y sus complicaciones en Medellín, Colombia, 2001-2003. Rev Panam Salud Publica. 2006;20(6):393-402.

13. Pinilla AE, Sánchez AL, Mejía A, del Pilar Barrera M. Primary-care prevention activities in outpatients suffering from diabetic foot care. Rev Salud Pública [Internet]. Instituto de Salud Publica, Faculdad de Medicina - Universidad Nacional de Colombia; [cited 2015 Aug 27];13(2):262-73. Available from: http://www.scielosp.org/scielo.php?script=sci_arttext\&pid=S012400642011000200008\&lng=en\&nrm=iso\&tlng.

14. Mythili A, Kumar KD, Subrahmanyam KA V, Venkateswarlu K, Butchi RG. A Comparative study of examination scores and quantitative sensory testing in diagnosis of diabetic polyneuropathy. Int J Diabetes Dev Ctries [Internet]. 2010 Jan [cited 2015 Aug 26];30(1):43-8. Available from: http://www. pubmedcentral.nih.gov/articlerender.fcgi?artid=2859284\&tool=pmcentre z\&rendertype $=$ abstract.

15. Peters EJ, Lavery LA. Effectiveness of the diabetic foot risk classification system of the International Working Group on the Diabetic Foot. Diabetes Care [Internet]. 2001 Aug [cited 2015 Aug 27];24(8):1442-7. Available from: http://www.ncbi.nlm.nih.gov/pubmed/11473084.

16. Júbiz Y, Brugés J, Orduz A, Bohórquez L, Calderón C, Díaz A, Escobar I, et al. Guías colombianas para la prevención, diagnóstico y tratamiento del pie diabético: un manejo integral [Internet]. 1st ed. Bogotá: COLPEDIS Grupo Colombiano de Pie Diabético; 2012.96 p. Available from: http://issuu.com/ presidenciafdc/docs/guias_pie_diabetico.

17. Jirkovská A, Bouček P, Wosková V, Bartoš V, Skibová J. Identification of patients at risk for diabetic foot: A comparison of standardized noninvasive testing with routine practice at community diabetes clinics. J Diabetes Complications. 2001;15(2):63-8.

18. Brownrigg JRW, Hinchliffe RJ, Apelqvist J, Boyko EJ, Fitridge R, Mills JL, et al. Effectiveness of bedside investigations to diagnose peripheral artery disease among people with diabetes mellitus: a systematic review [Internet]. London: International Working Group on the Diabetic Foot;
2015. p. 26. Available from: http://www.iwgdf.org/files/2015/PADdiagnosis.pdf

19. Driver VR, Fabbi M, Lavery LA, Gibbons G. The costs of diabetic foot: the economic case for the limb salvage team. J Vasc Surg [Internet]. Elsevier; 2010 Sep 9 [cited 2015 Apr 21];52(3 Suppl):17S - 22S. Available from: http:// www.jvascsurg.org/article/S0741521410013248/fulltext.

20. Tsourdi E, Barthel A, Rietzsch H, Reichel A, Bornstein SR. Current aspects in the pathophysiology and treatment of chronic wounds in diabetes mellitus [Internet]. BioMed Research International. 2013. p. 6. Available from: http://dx.doi.org.10.1144/2013/385641.

21. Lipsky BA, Berendt AR, Cornia PB, Pile JC, Peters EJG, Armstrong DG, et al. 2012 infectious diseases society of America clinical practice guideline for the diagnosis and treatment of diabetic foot infections. Clinical Infectious Diseases. 2012. p. 1679-84.

22. Monteiro-Soares M, Boyko EJ, Ribeiro J, Ribeiro I, Dinis-Ribeiro M. Predictive factors for diabetic foot ulceration: A systematic review. Diabetes/Metabolism Research and Reviews. 2012. p. 574-600.

23. American Diabetes Association. Microvascular complications and foot care. Diabetes Care [Internet]. 2015;38(Suppl 1):S58-66. Available from: http:// care.diabetesjournals.org/content/38/Supplement_1/S58.full.

24. Dorresteijn J a N, Kriegsman DMW, Valk GD. Complex interventions for preventing diabetic foot ulceration. Cochrane Database Syst Rev. 2010;(1):CD007610.

25. Dorresteijn JAN, Kriegsman DMW, Assendelft WJJ, Valk GD. Patient education for preventing diabetic foot ulceration. Cochrane database Syst Rev [Internet]. 2014 Jan [cited 2015 Jul 28];12:CD001488. Available from: http:// www.ncbi.nlm.nih.gov/pubmed/25514250.

26. National Institute for Health and Care Excellence. Diabetic foot problems: prevention and management [Internet]. 2015. 46 p. Available from: nice. org.uk/guidance/ng19.

27. Fan L, y col , Feasibility, Acceptability and Effects of a Foot Self-Care Educational Interventionon Minor Foot Problems in Adult Patients with Diabetes at Low Risk for Foot Ulceration: A Pilot Study . Canadian journal of diabetes. March 2013. journal homepage:www.canadianjournalofdiabetes.com

28. Corbett C. y col . A rambdomized pilot study of improving foot care in home health patients with diabetes. The diabetes educator, vol 29 ,number 2 March 2003. 